\title{
Architecture and Performance Evaluation of a Novel Optical Packet Switch with Input Concentrators
}

\author{
Hongzhen Yang ${ }^{1}$, Xiuwei Mao ${ }^{1}$, Zilu Fang ${ }^{1}$, Wanke Chen ${ }^{1}$, Ting Wang ${ }^{1}$, Shuna Yang ${ }^{2}$, Yuhu Zhao ${ }^{2}$ \\ and $\mathrm{Hao} \mathrm{Chi}^{2, *}$ \\ 1 Information and Telecommunication Branch, State Grid Zhejiang Electric Power Co., Ltd., Hangzhou 310020, \\ China; yang_hongzhen@zj.sgcc.com.cn (H.Y.); mao_xiuwei@zj.sgcc.com.cn (X.M.); \\ fang_zilu@zj.sgcc.com.cn (Z.F.); chen_wanke@zj.sgcc.com.cn (W.C.); wangting_xt@zj.sgcc.com.cn (T.W.) \\ 2 School of Communication Engineering, Hangzhou Dianzi University, Hangzhou 310018, China; \\ shunayang@hdu.edu.cn (S.Y.); ztiger62@hdu.edu.cn (Y.Z.) \\ * Correspondence: chihao@hdu.edu.cn
}

Citation: Yang, H.; Mao, X.; Fang, Z.; Chen, W.; Wang, T.; Yang, S.; Zhao, Y.; Chi, H. Architecture and Performance Evaluation of a Novel Optical Packet Switch with Input Concentrators. Photonics 2021, 8, 510. https:// doi.org/10.3390/photonics 8110510

Received: 12 September 2021 Accepted: 10 November 2021 Published: 12 November 2021

Publisher's Note: MDPI stays neutral with regard to jurisdictional claims in published maps and institutional affiliations.

Copyright: (c) 2021 by the authors. Licensee MDPI, Basel, Switzerland. This article is an open access article distributed under the terms and conditions of the Creative Commons Attribution (CC BY) license (https:// creativecommons.org/licenses/by/ $4.0 /)$

\begin{abstract}
In this paper, we propose a novel optical packet switch (OPS) architecture with input concentrators, which employ multi-input single-output optical buffers to aggregate all the incoming traffic into a small size switching fabric. Accordingly, the physical size, the number of the needed wavelength converters, and the economic cost of the total OPS node are decreased dramatically. However, the deployment of input concentrators introduces additional packet loss and delay, except from the contention at the switch output. A Markov model is presented to study the packet loss ratio (PLR) and average packet delay given by the input concentrators. The corresponding closed form expressions are given. The model also demonstrates that the system performance can be greatly improved by increasing the buffer size when the traffic load is not larger than 0.69315 . The analytical values are compared with the simulation results. All the obtained results show that the proposed model provides satisfactory approximations under different network scenarios. Moreover, the economic cost savings of the proposed OPS node at the present time and its evolution as a function of time are also discussed in detail. The proposed architecture can also be applied in a packet enhanced optical transport network (OTN).
\end{abstract}

Keywords: optical packet switch (OPS); Markov model; fiber delay line (FDL); packet loss ratio (PLR); average packet delay; packet enhanced optical transport network (OTN)

\section{Introduction}

Driven by increasing communication needs worldwide, next generation optical networks are expected to provide huge bandwidth as well as support for diverse service demands. These requirements can be fulfilled by the high capacity offered by the next generation optical transport network (OTN) [1]. To fully utilize the provided capacity, different kinds of switching paradigms in the optical domain are employed to eliminate the processing bottleneck in the electronic domain. A lot of attention has been paid to hybrid approaches [2,3], which combine optical circuit switching (OCS) with optical packet switching (OPS) and/or optical burst switching (OBS) to support different QoS demands. In this paper, we focus on the OPS part of such hybrid architectures, which is used to switch the lower bandwidth demand service class. Since OPS has smaller switching granularity and much more immature production technology compared with OCS, its architecture would dominate the total physical size and economic cost of the hybrid switching node.

In the past, various OPS architectures have been proposed, and their performance evaluations have been done in terms of the packet loss ratio (PLR) and average packet delay. Refs. [4,5] described and evaluated the performance of the broadcast and select OPS architecture proposed in the European Advanced Communication Technologies and Services (ACTS) KEOPS (keys to optical packet switching). Ref. [6] proposed a scalable 
design for OPS architecture with two stages of tunable wavelength converters (TWCs), and its performance was analyzed in detail. However, most of the proposed OPS architectures are mainly focused on the packet contention resolution (optical buffering, properly designed fiber delay lines, deflection routing) [7-10], fast packet switching (device, packet synchronization) [11], and QoS [12]. Not much attention is paid regarding the physical size and the economic cost, which are also major issues for the OPS architecture.

In this paper, we proposed a novel OPS architecture with input concentrators, which are composed of fixed length fiber delay lines (FDLs) and work as multi-input single-output optical buffers. According to the input wavelength, the specific concentrator aggregates the incoming traffic from all input fibers into its respective output link, which is followed by the TWC and the input channel of the switching fabric. Correspondingly, the physical size, the number of needed TWCs, and the economic cost of the total OPS node are reduced dramatically. In addition, the incoming traffic pattern will be smoothed significantly by the concentrator, leading to very small and negligible packet loss from the contention at the switch output. However, the deployment of input concentrators introduces additional packet loss and delay from the traffic aggregation. In this paper, we present a Markov analytical model to study the input PLR and the average packet delay introduced by input concentrators. The corresponding closed form expressions are given. The model also demonstrates that the buffer size provided by each concentrator will be used up when the total incoming traffic load is larger than 0.69315 . This analytical model is validated by the simulations under different network scenarios.

The proposed OPS architecture realizes having a smaller physical size, needing fewer TWCs, a higher link utilization, and a lower economic cost. Therefore, it will play a very important role in a number of network scenarios. As mentioned above, it can improve some hybrid optical switch architectures like OpMiGua, "Optical Migration Capable Networks with Service Guarantees" [13], which uses OPS to switch the lower bandwidth demand's service classes. Since only a small amount of incoming traffic are mapped into this kind of service class, our proposed OPS architecture can be used for handling them and to realize the more compact, lower economic cost, and smaller physical size of the total hybrid switch architecture. The proposed OPS architecture can also be used in the access network, which is responsible for collecting end-users' traffic and has a higher demand of the granularity and flexibility. The deployment of the proposed OPS node will lead to higher link utilization, larger cost saving, higher transmission speeds, smaller physical size, and more flexibility for the access network. In addition, the proposed architecture of the concentrator can also be used for other network scenarios, like ORION (overspill routing in optical networks) [14] and packet enhanced OTN [15]. Ref. [16] proposed the constrained ORION and pointed out the considerable advantages compared with the unconstrained ORION. However, the detailed architecture of how to implement the concentration is not described. The proposed concentrator is a good candidate for this.

The rest of this paper is organized as follows. We present the novel OPS node, the detailed architecture, and the operation of the employed concentrator in Section 2. Section 3 introduces the analytical model and presents the closed form expressions of both the PLR and the average packet delay. The results from both simulation and the analytical model are shown in Section 4. Finally, our conclusions are given in Section 5.

\section{OPS Structure with Concentrator}

\subsection{The Proposed OPS Architecture}

Figure 1 shows the proposed OPS architecture with input concentrators. This architecture consists of $F$ input/output fibers, each capable of carrying $W$ independent wavelengths. After being separated by the demultiplexer, the same channel/wavelength $\lambda_{i}$ from all input fibers are connected to one specific concentrator $(1 \leq i \leq W)$, which aggregates the total incoming traffic into one output link. This link is followed by the WC and the input port of the switching fabric. Correspondingly, all the incoming traffic from $F \times W$ input channels are concentrated into $W$ channels and handled by small size $(W \times W)$ switching fabric. 
Hence, the physical size and the needed WCs of the whole OPS architecture are decreased significantly. The detailed architecture and the operation of the concentrator is given in part $B$ in this section.

In contrast to the OPS architecture without input concentrators, the proposed architecture has several significant improvements. First of all, the physical size of the total OPS architecture is reduced dramatically. Consider the OPS without concentrator, $F$ input fibers with $W$ wavelengths per fiber lead to $F \times W$ input channels, which need a $(F \times W) \times(F \times W)$ switching fabric to handle all the traffic. However, for the proposed architecture, all the incoming traffic have to go through the input concentrators before being sent to the switching fabric, hence a $(W \times W)$ switching fabric is enough for $W$ wavelength per fiber, independent of the number of the input fibers. Furthermore, the number of the needed WCs is decreased significantly. For WC, which is necessary for supporting the routing path choice in OPS, its number is same as that of the input channels of the employed switching fabric. Hence, it will decrease as the size of switching fabric decreases. In addition, the total economic cost of the OPS structure is considerably saved. Since both WC and switching fabric dominate the economic cost of the total OPS node, it will be largely saved in case both the number of WCs and the physical size of the switching fabric decrease.

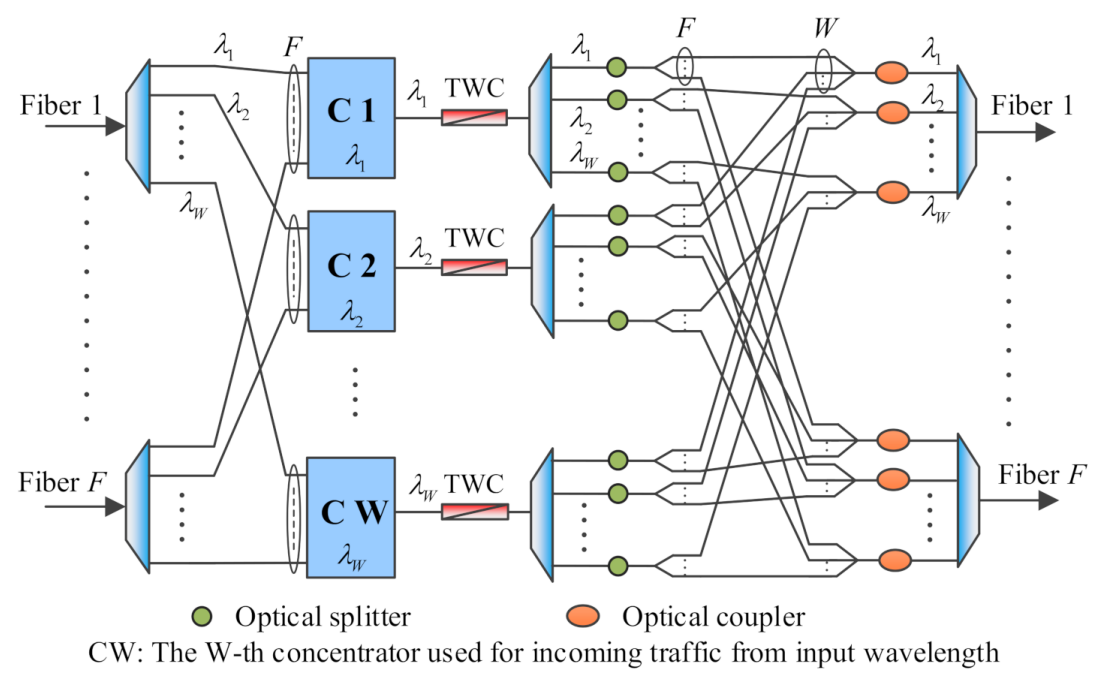

Figure 1. The proposed OPS architecture with input concentrators.

\subsection{The Architecture and Operation of the Input Concentrator}

The detailed architecture of the employed input concentrator is shown in Figure 2a. It consists of two parts: the space switch and the optical buffers. The former is used for choosing the suitable inserted position for the arriving packet. The latter provides the respective delay according to the inserted position. Different from the general optical buffer, the optical buffer here is realized by the same FDL elements which are connected in sequence. Each FDL element is connected to the next one by an optical coupler. Although different arriving packets might be inserted into different positions, in the end all of them will be sent out by the same output port. Hence, the concentrator works like a multiple-input single-output optical buffer. Note that we assume the optical coupler has negligible loss.

Figure $2 \mathrm{~b}$ gives the detailed operation description of the concentrator for the arriving packet. Due to the possible input ports of the optical buffers is $(K+1)$ (from 0 to $K$ ), the whole concentrator can be considered as a $(K+1)$-positions queue. The solid arrows denote all possible inserted positions for the arriving packet. When the packet arrives, it will check the current queue length and choose the available inserted position. Note that in order to avoid the collision with the packet already transmitted in queue, the current queue length is equal to the largest index of the occupied inserted positions. For instance, 
if position 4 is busy transmitting one packet, even if position 2 is empty, the current queue length is still 4 , and the new arriving packet can only be inserted in position 5 but not any position with a smaller index.

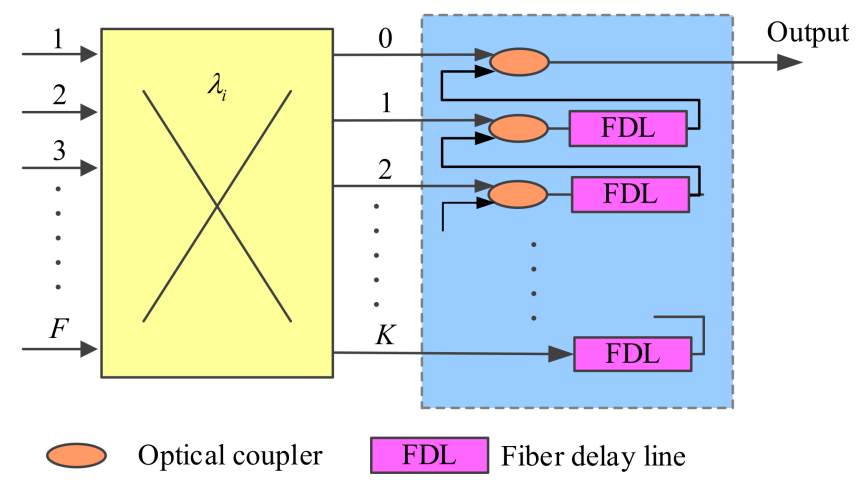

(a)

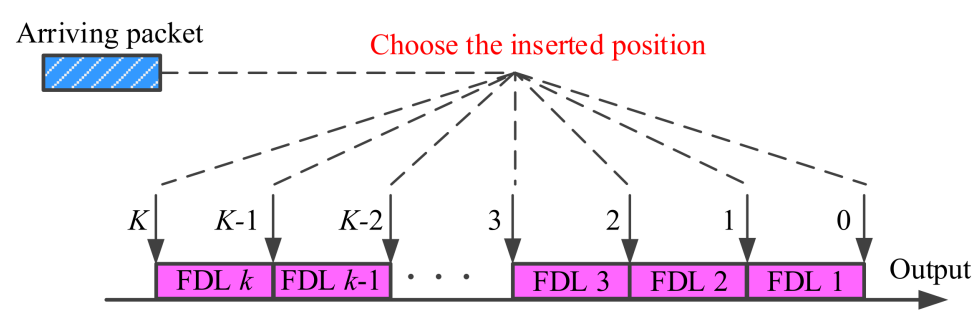

(b)

Figure 2. (a) The detailed architecture of the concentrator. (b) Operation of the concentrator for arriving packets.

\section{Analytical Model}

In this section, we study the performance of the proposed OPS structure. Due to the incoming traffic pattern having been smoothed heavily by input concentrators, the PLR introduced by the contention at the switch output is very small and can be neglected. In the following analysis, we study the PLR and the average packet delay given by input concentrators. A Markov analytical model is built based on the following assumptions:

- Uniform traffic pattern for each input fiber, i.e., each input fiber gives the equal traffic load);

- Uniform wavelength distribution of the incoming requests, i.e., each incoming wavelength gives the same traffic load). We can restrict our study to only one incoming wavelength from different input fibers;

- The Poisson arrival processes for the packets from each input wavelength, i.e., a sufficient large number of flows are aggregated in core network to allow the Poisson arrival process for the packets carried by each input wavelength;

- The packet length is fixed, and its size is same as the FDL length. In this paper, we consider the fixed length packets, which is the case for most proposed asynchronous OPS networks [17].

As described in Section 2, the same wavelengths from all input fibers are connected to one corresponding input concentrator which works as a multiple-input single-output optical buffer. Since the packet arrives following the Poisson process, the inter-arrival time between the consecutive packets follows a negative exponential distribution (n.e.d). We assume the packet arrival intensity per wavelength is $\lambda$, the service time for each packet is $1 / \mu$. Note that due to the packet size being the same as the FDL length, the service time for each packet is the same as the delay time provided by one FDL element. In the following analysis, we restrict our study to only one input concentrator. 
Figure 3 shows a timing paradigm to present the detailed packet arrival and departure process from the studied concentrator. PCK $i$ above each packet denotes the $i$ th arriving packet to the studied concentrator. FDL $i$ denotes the $i$ th FDL element the respective arriving packet should go through. The number of FDLs denotes the delay time of this packet introduced by the concentrator. For PCK $i$, its delay is equal to $(\mathrm{T} i-\mathrm{t} i-1 / \mu)$. t $i$ denotes the arriving time and $T i$ denotes its leaving time out of the concentrator. $Q$ in the left of the timing paradigm indicates the current queue length (from 0 to $K$ ) just after the packet arrives in the concentrator. Its value depends on the current inter-arrival time $(\Delta t)$ with this new arriving packet. Pij indicates the transition probability from the state $Q=i$ to the state $Q=j$. The bottom of the timing diagram presents the packets departure process from the concentrator. Hence, the link utilization can be obtained by this departure process.

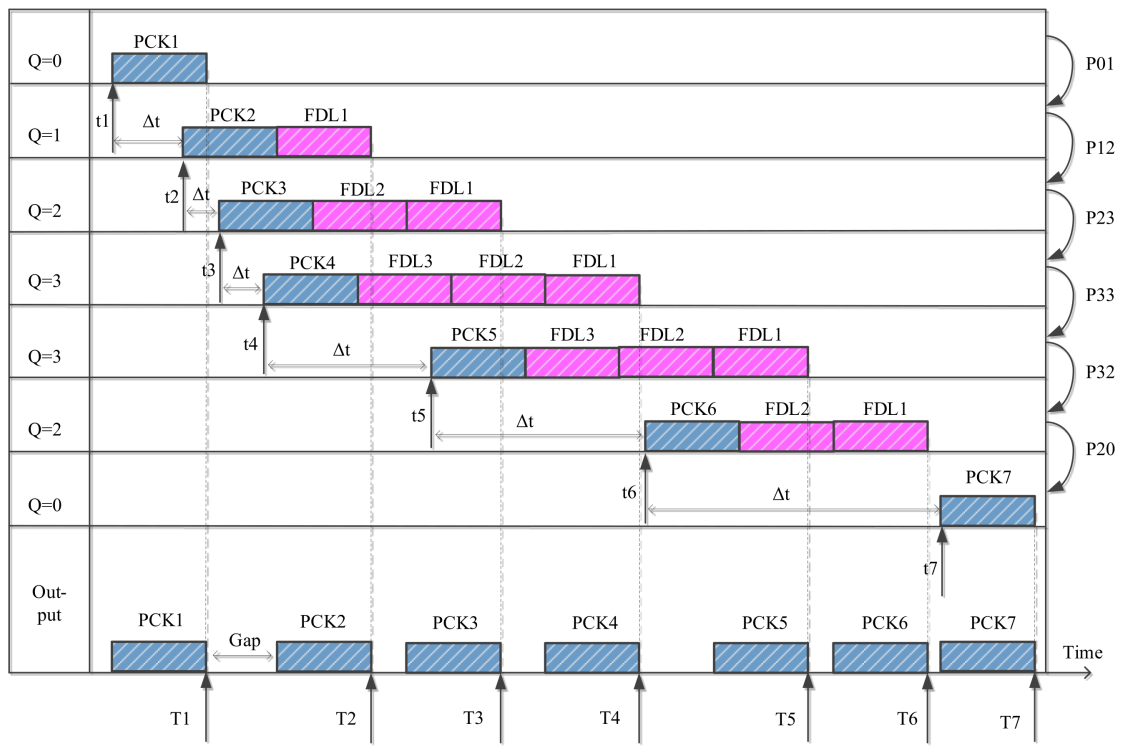

Figure 3. An example timing diagram of the packet departure process from the studied concentrator structure.

We can explain Figure 3 in the following way. The concentrator works like a memoryless optical buffer. The queue length's evolution depends on the current queue length and the current inter-arrival time $(\Delta t)$ with the new arriving packet. If $0 \leq \Delta t<1 / \mu$, the new packet arrives before the previous one finishes its transmission into the concentrator. The current queue length $Q$ will increase by 1 . In Figure 3, from $Q=0$ to $Q=3$, once the $\Delta t$ is smaller than $1 / \mu, Q$ increases by 1 . If FDL $\leq \Delta t<2 \times$ FDL, when the new packet arrives, the previous packet has transmitted into the concentrator, however it has not finished its transmission time through one FDL element. Accordingly, the current queue length will not change. Using the same method, if $2 \times \mathrm{FDL} \leq \Delta t<3 \times \mathrm{FDL}$, the queue length will decrease by 1 . If $n \times \mathrm{FDL} \leq \Delta t<(n+1) \times \mathrm{FDL},(n>1)$, the queue length will decrease $(n-1)$. In Figure 3, when PCK 7 arrives, $3 \times$ FDL $<\Delta t<4 \times$ FDL, the PCK 6 has already gone through the 2 th, 1 th, and the 0 th position in the queue, so PCK 7 will be inserted in the 0th position in the current queue and the $Q$ decreases by 2 and becomes 0 .

According to the discussion above, the Markov model is built as shown in Figure 4 . 


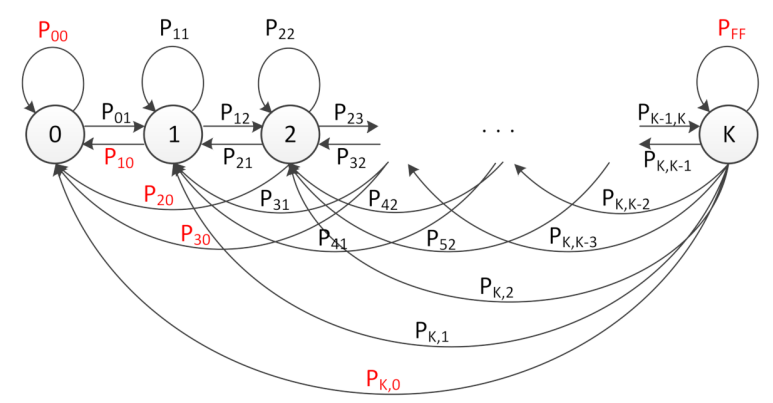

Figure 4. Markov model state transitions for the studied concentrator.

The maximum state is $K$, which denotes the state that the current queue length is $K$, and if the new packet arrives on this state, this packet will be dropped directly.

In order to get the needed PLR and average packet delay from Figure 4, we have to calculate the probability of each state and the transition probabilities among them.

Next, we derive the transition probabilities in Figure 4 . If $0 \leq \Delta t<1 / \mu$, the value of $Q$ will increase by 1 . The packet arrival process follows the Poisson process, hence:

$$
P_{i(i+1)}=p(0 \leq t<1 / \mu)=\int_{t=0}^{t=1 / \mu} \lambda e^{-\lambda t}=1-e^{-A}
$$

where $=\lambda / \mu$.

If $1 / \mu \leq \Delta t<2 \times 1 / \mu$, the value of $Q$ will not change, so:

$$
P_{i i}=p(1 / \mu \leq t<2 / \mu)=\int_{t=1 / \mu}^{t=2 / \mu} \lambda e^{-\lambda t}=e^{-A}-e^{-2 A}
$$

For $(1 \leq i \leq K-1), P_{i i}$ is equal to $\left(e^{-A}-e^{-2 A}\right)$. Note that when $i=0, K$, the calculation for the probability is different. When $i=0$, the current queue length is smallest as 0 , the state will not change unless $\Delta t$ is smaller than $1 / \mu$. Its probability is:

$$
P_{00}=1-p(0 \leq t<1 / \mu)=1-\int_{t=0}^{t=1 / \mu} \lambda e^{-\lambda t}=e^{-A}
$$

For $i=K$, the current queue length is largest as $K$, the state will not change unless $\Delta t$ is larger than $2 \times 1 / \mu$, which leads to the current queue length decreasing, and the state returns to other smaller states. Otherwise, if $0 \leq \Delta t<2 \times 1 / \mu$, the state keeps same, and the new arrival packet is dropped directly. Hence its probability is:

$$
P_{K K}=1-p(t>2 / \mu)=p(0 \leq t<2 / \mu)=1-e^{-2 A}
$$

If $2 \times 1 / \mu \leq \Delta t<3 \times 1 / \mu$, the current queue length will be decreased by 1 . Hence:

$$
P_{i(i-1)}=p(2 / \mu \leq t<3 / \mu)=e^{-2 A}-e^{-3 A}
$$

For $1 \leq i \leq K, P_{i(i-1)}$ is equal to $\left(e^{-2 A}-e^{-3 A}\right)$. Note that $P_{10}$ is different, if $\Delta t$ is larger than $2 \times 1 / \mu$, the current state 1 will go back to the state 0 . Hence:

$$
P_{10}=p(t>2 / \mu)=1-p(0<t<2 / \mu)=e^{-2 A}
$$


If $n \times 1 / \mu \leq \Delta t<(n+1) \times 1 / \mu,(n>1)$, the current queue length will decrease $(n-1)$. So:

$$
P_{i(i-n+1)}=p(\mathrm{n} / \mu \leq t<(\mathrm{n}+1) / \mu)=e^{-n A}-e^{-(n+1) A}
$$

For $(1 \leq i \leq K), P_{i(i-n+1)}$ is equal to $\left(e^{-n A}-e^{-(n+1) A}\right)$. Note that $P_{i 0}$ is different, if $\Delta t$ is larger than $(i+1) \times 1 / \mu$, the current state $i$ will go back to the state 0 . Hence:

$$
P_{i 0}=p(t>(\mathrm{i}+1) / \mu)=e^{-(i+1) A}
$$

According to the derived transition probabilities and the node equations, the probability of each state can be obtained. In the following formulas, we use $\pi(i)$ to denote the probability of state $i$. The node equations are listed as follows:

$$
\left\{\begin{array}{c}
\pi(0) \times P_{01}=\sum_{i=1}^{i=K} \pi(i) \times P_{i 0}, \quad 1 \leq i \leq K \\
\pi(K) \times \sum_{j=0}^{j=K-1} P_{K j}=\pi(K-1) \times P_{(K-1) K} \\
\pi(i) \times\left(P_{i(i+1)}+\sum_{j=0}^{j=i-1} P_{i j}\right)=\pi(i-1) \times P_{(i-1) i}+\sum_{j=i+1}^{j=K} \pi(j) \times P_{j i} \\
\sum_{i=0}^{i=K} \pi(i)=1
\end{array}\right\}
$$

Submitting transition probabilities into the node equations above, the probability of each state can be derived as:

$$
\pi(i)=\left\{\begin{array}{cl}
\sum_{j=0}^{K} \pi(j) \times e^{-(j+1) A}, & i=0 \\
\sum_{j=1}^{K} \pi(j) \times\left[e^{-(j-i+1) A}-e^{-(j-i+2) A}\right], & 1 \leq i \leq K
\end{array}\right\}
$$

The solution can be obtained as:

$$
\pi(i)=\frac{D^{i} \times(1-D)}{1-D^{K+1}}, \quad 0 \leq i \leq K
$$

where $D=e^{A}-1$. Hence, $\pi(K)=\pi(0) \times D^{K}$. As discussed in [5], only when $D<1$, the Markov chain is positive recurrent and has stationary distribution. If $D<1$ :

$$
e^{A}-1<1 \Rightarrow A<\ln 2 \Rightarrow A<0.69315
$$

Therefore, we can conclude that no matter how large the buffer size provided by each concentrator is, it will be used up when the total incoming traffic load is larger than 0.69315 .

In order to calculate the studied PLR, first we have to find the difference between the electronic buffer and the optical buffer. For the electronic buffer, when the queue length is $K$, all the positions in the queue are occupied, then all the new incoming packets will be dropped directly. Hence, for the electronic buffer, the packet loss probability is equal to $\pi(K)$. However, for the optical buffer, the new arriving packet still can be served if $\Delta t$ (the current inter-arrival time) is larger than $1 / \mu$ and smaller than $2 \times 1 / \mu$ $(1 / \mu \leq \Delta t<2 \times 1 / \mu)$. Therefore, the packet loss only happens when the current interarrival time is smaller than $1 / \mu$ while the current queue length is $K$. Note that when $K=0$, the studied concentrator has no FDL element to provide time delay, and all the incoming packets will be sent out or dropped directly. The studied concentrator can be considered as an $\mathrm{M} / \mathrm{M} / 1$ system, and the corresponding PLR can be obtained by the Erlang loss formula. Combined with Equation (1), the closed form of the studied PLR is:

$$
\left\{\begin{array}{c}
P L R=\pi(K) \times p(0 \leq t<1 / \mu)=\frac{(1-D) D^{K+1}}{(1+D)\left(1-D^{K+1}\right)}, \quad K>0 \\
P L R=\frac{A}{1+A}, \quad K=0
\end{array}\right\}
$$


According to Figure 4, we also get the average packet delay for all the transmitted packets. For instance, state $Q$ denotes the current queue length, and it is same as the inserted position of the respective packet. Hence, for state $Q$, the delay time for the inserted packet is $(Q-1) \times 1 / \mu$. Note that when $K=0$, all the incoming packets to the concentrator will be sent out or dropped directly. The average packet delay is 0 . We use $\bar{D}$ to denote the average delay time, and its closed form expression is:

$$
\left\{\begin{array}{c}
\bar{D}=\sum_{i=0}^{K} \pi(i) * \frac{(i-1)}{\mu}=\sum_{i=0}^{K} \frac{D^{i} *(1-D)(i-1)}{\left(1-D^{K+1}\right) \mu}, \quad K>0 \\
\bar{D}=0, \quad 00
\end{array}\right\}
$$

\section{Simulation and Analytical Results}

A simulation model for an asynchronous OPS node equipped with input concentrators is built in the discrete event modeling on Simula (DEMOS) software. Ten independent simulations were performed for each parameter setting. For all the simulation results, we calculated the average value. All the analytical results are obtained by Equations (11), (13), and (14). Table 1 summarizes all the employed variables in our simulations. The "values" in Table 1 present all possible used values under the different scenarios.

Table 1. The parameters and the corresponding values.

\begin{tabular}{ccc}
\hline Para & Description & Values \\
\hline$F$ & Number of input/output fibers & 4 \\
$w$ & Number of wavelengths per fiber & 8 \\
$C$ & Capacity of each wavelength & $10 \mathrm{Gbps}$ \\
$A$ & Normalized system load per wavelength & $0.05 \leq A \leq 0.2$ \\
$S$ & Total incoming traffic load per concentrator & $F \times A$ \\
$M$ & Mean packet size & $800 \mathrm{bits}$ \\
$K$ & Number of FDLs per concentrator & $0 \leq K \leq 75$ \\
\hline
\end{tabular}

For simulations, we consider one Poisson source which generates packets into the proposed OPS node. For the case where the incoming packets have a fixed length, the packet size is fixed as 800 bits. For the case where the incoming packets have a variable length, the packet size follows n.e.d. with the mean value of 800 bits. The link capacity is set as $10 \mathrm{Gbps}$. Accordingly, the mean service time for both cases is equal to 800 (bits) $/ 10$ (Gbps) = $0.8 \times 10^{-7}$ (s) (i.e., $1 / \mu=0.8 \times 10^{-7}$ ). The packet inter-arrival time for both cases follows n.e.d. Its mean value $1 / \lambda$ is set according to the total traffic load (A) and $\lambda=A \times \mu$. In this section we will give the results for the two cases separately.

Case I: The incoming packets have fixed length

In a network with fixed length packets, a natural choice for the buffer is to let the basic delay line map the packet length. In this part we set the length of the basic delay line element in the input concentrator as the same as the packet length to evaluate its performance.

For the proposed OPS node, the channels with the same wavelength from all different input fibers are connected to the specified input concentrator. For each input concentrator, when the packet arrives, it will check the current queue length inside the concentrator. If the queue length is equal to zero, the packet will be sent out directly, otherwise the packet will choose the suitable inserted position and will be sent out by passing through the respective delay time. In the case that the last position of the queue is occupied, this new arriving packet is dropped out directly.

Figure 5 presents the input PLR of the system as a function of the normalized incoming traffic load arrived per concentrator with different configurations, i.e., the buffer size or the FDL number per concentrator is changed from 0 to $75(0 \leq K<75)$. Here the traffic 
load denotes the total incoming traffic load for each concentrator. It is equal to $F \times A$ (the number of input fibers times the normalized system load per wavelength).

Results from both the analytical model and simulations are shown. S. denotes the results from the simulation model and A. indicates the results from the built analytical model. The main observation is that the analytical values approximate the simulation results very well under different network scenarios.

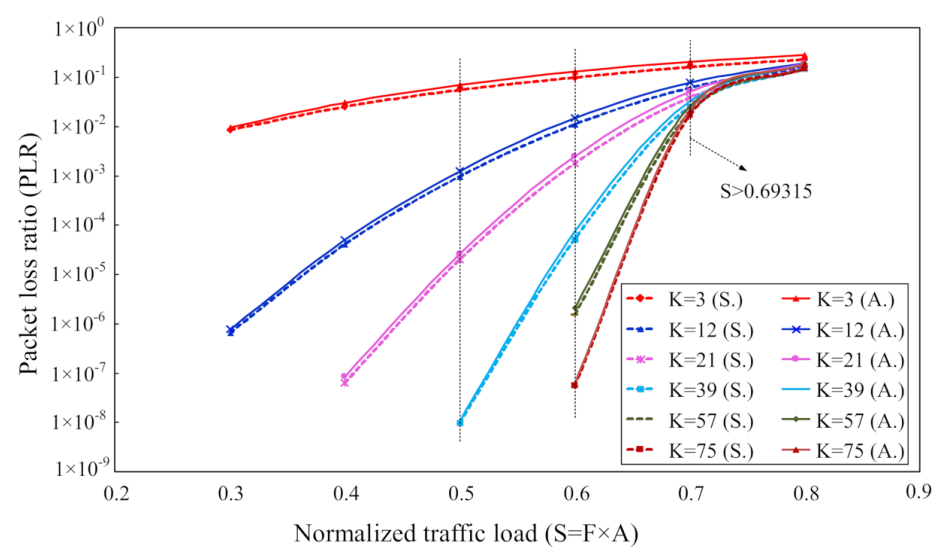

Figure 5. Packet loss ratio versus normalized incoming traffic load per concentrator.

A number of observations can be achieved from Figure 5. When the number of FDL elements is equal to 0 , all the arriving packets will be sent out or dropped directly; the studied concentrator can be considered as an M/M/1 system; the PLR is obtained by the Erlang loss formula directly. Figure 5 shows the PLR under a different incoming traffic load per concentrator. As expected, under the fixed traffic load, which is not larger than 0.69 , i.e., $S<0.6935$, the PLR of whole system decreases very fast as the buffer size increases. For instance, when the normalized traffic load is 0.5 , the PLR is about $1.0 \times 10^{-3}$ with the number of FDL elements is 12 , and it decreases to $1.0 \times 10^{-8}$ when $\mathrm{K}$ increases to 39 . Hence, the system performance can be greatly improved by increasing the number of FDL elements under the low traffic loads. However, as with the discussion given in Section 3, the buffer size provided by each concentrator will be used up when the total incoming traffic load is larger than 0.69315 . This means that when the traffic load is larger than 0.69315 , the concentrator can be considered as an overflow system, and its performance cannot be improved even with the larger buffer size. As shown in Figure 5, when the total system load is 0.7 , the obtained PLR values are very close if the number of FDL elements is larger than 39, which means that the improvement of the system performance is very limited by increasing the buffer size per concentrator. When the traffic load is 0.8 , the system PLR values are equal when the number of FDL elements is larger than 21, which means the buffer size is used up and cannot be improved by simply increasing the FDL elements under the high traffic load. To sum up, for the proposed OPS architecture with input concentrators, we can conclude that when the traffic load is small, and especially smaller than 0.69315 , the PLR can be reduced significantly by adding the number of FDL elements in each concentrator. However, for a large traffic load, the addition of the FDL elements per concentrator cannot improve the performance while leading to a longer average packet delay.

Figure 6 shows the average packet delay of the system as a function of the normalized incoming traffic load under the different buffer sizes provided per concentrator. As in Figure 5, the traffic load denotes the total incoming traffic load per concentrator. It is also equal to $F * A$, as shown in Table 1. The analytical results are obtained by Equation (3). Compared with the simulation results, we can conclude that the analytical values are in good agreement with the simulation results, which validates the correctness and the feasibility of the proposed analytical model. 
When the number of FDL elements is equal to 0 , i.e., $K=0$, the arriving packet will be sent out or dropped directly, and no delay exists, which is not given in Figure 6. We can see that the average packet delay increases as the traffic load grows up under the fixed buffer size. For instance, when the number of FDL elements is fixed as 12, the average packet delay is smaller than $1.0 \times 10^{-7}$ when the traffic load is 0.3 and becomes very close to $1.0 \times 10^{-6}$ under the traffic load of 0.8 . Furthermore, when the traffic load is smaller than 0.69315, we can see that, as the FDL number increases, the average packet delay increases very fast at first and becomes quite stable by increasing the buffer size. As shown in Figure 6, when the traffic load is 0.4 , the average delay can be improved by increasing the buffer size and becomes stable when the FDL number is larger than 12 . When the traffic load is 0.5 , the average delay cannot be improved when $K \geq 21$. When the traffic load is 0.6 , the average delay becomes stable under $K \geq 39$. Combined with the results shown in Figure 5, which validates that the PLR can be reduced dramatically as the FDL number increases, we can conclude that for the low incoming traffic load, increasing the FDL number can improve the performance of the studied OPS structure while no additional delay will be introduced. However, when the traffic load is larger than 0.69315 , the buffer size will be used up no matter how large the buffer size provided by each concentrator, hence the average packet delay will increase and cannot be stationery for the large traffic load. As shown in Figure 6, when the system load is 0.7 and 0.8, the average packet delay does not have the threshold value from which the delay improvement is marginal. This can be explained by the difference between the electronic buffer and the optical buffer. For the electronic buffer, the buffered packets can be transmitted back-to-back, and no voids exist between two consecutive packets. The electronic buffer will be used up when the system load is larger than 1 . However, for the optical buffer, as discussed in Section 3, due to the voids between any two consecutive transmitted packets, the buffer size will be used up when the system load is larger than 0.69315 . Hence, we can conclude that for the large traffic load larger than 0.69315 , we cannot improve the performance of the studied OPS structure by adding the number of FDL elements per concentrator.

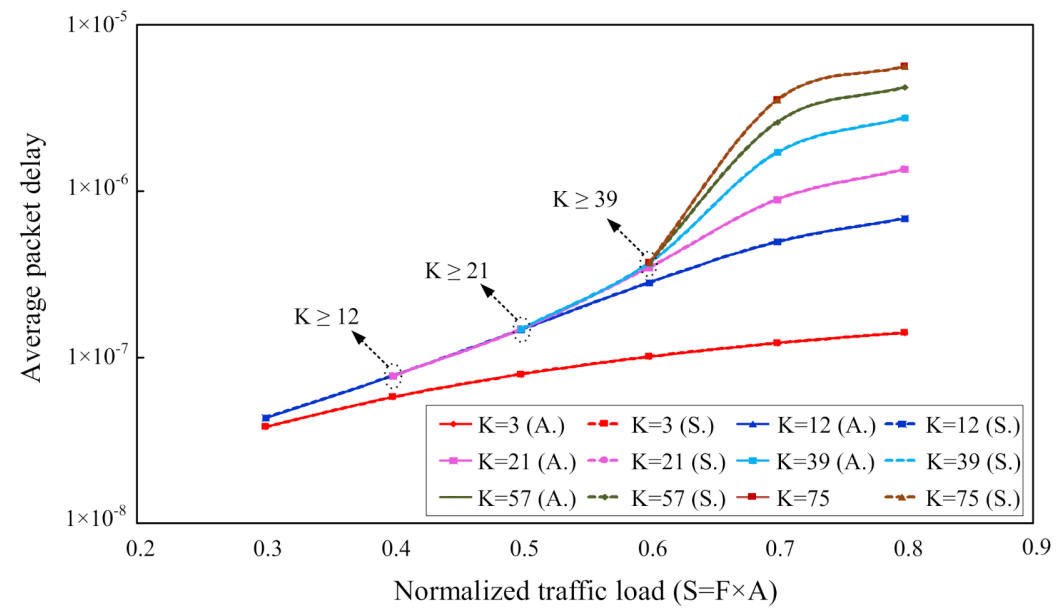

Figure 6. Average packet delay versus the normalized incoming traffic load per concentrator.

Case II: The incoming packets have variable length

Here we consider the incoming packets following n.e.d. with the mean packet size of 800 bits. The length of the basic delay line element is set as the same as the mean packet size. Therefore, the delay time offered by each delay line element is same as the mean packet service time (i.e., $d=1 / \mu$ ).

Figure 7 presents the $P L R$ values and the average packet delay per input concentrator as the function of the offered buffer size $(K>0$, i.e., the number of FDL elements). All simulation results are given under different system loads. As can be seen from Figure 7a, 
when traffic load $S \leq 0.6$, as buffer size $K$ increases, the PLR values decrease very fast. Accordingly, the network performance can be improved significantly by increasing the buffer size under low traffic loads. Figure $7 \mathrm{~b}$ shows that as $K$ increases the average packet delay time increases very fast at first and then becomes stable after one turning point. Therefore, we can conclude that increasing $K$ value (i.e., increasing the buffer size) will improve the performance of the optical buffer dramatically under the low system loads $(S \leq 0.6)$ : the PLR value decreases significantly without any additional delay time. However, when traffic load $S \geq 0.7$, as $K$ increases, the PLR values decrease very fast at first and become stable after one turning point. Moreover, the average packet delay value increases very fast as $K$ increases. No threshold after which the delay time will become stable exists. Thus, we can conclude that the performance of the optical buffer cannot be improved by increasing the buffer size under high traffic loads $(S \geq 0.7)$ : the $P L R$ value cannot be reduced while the packet delay value increases as the buffer size grows.
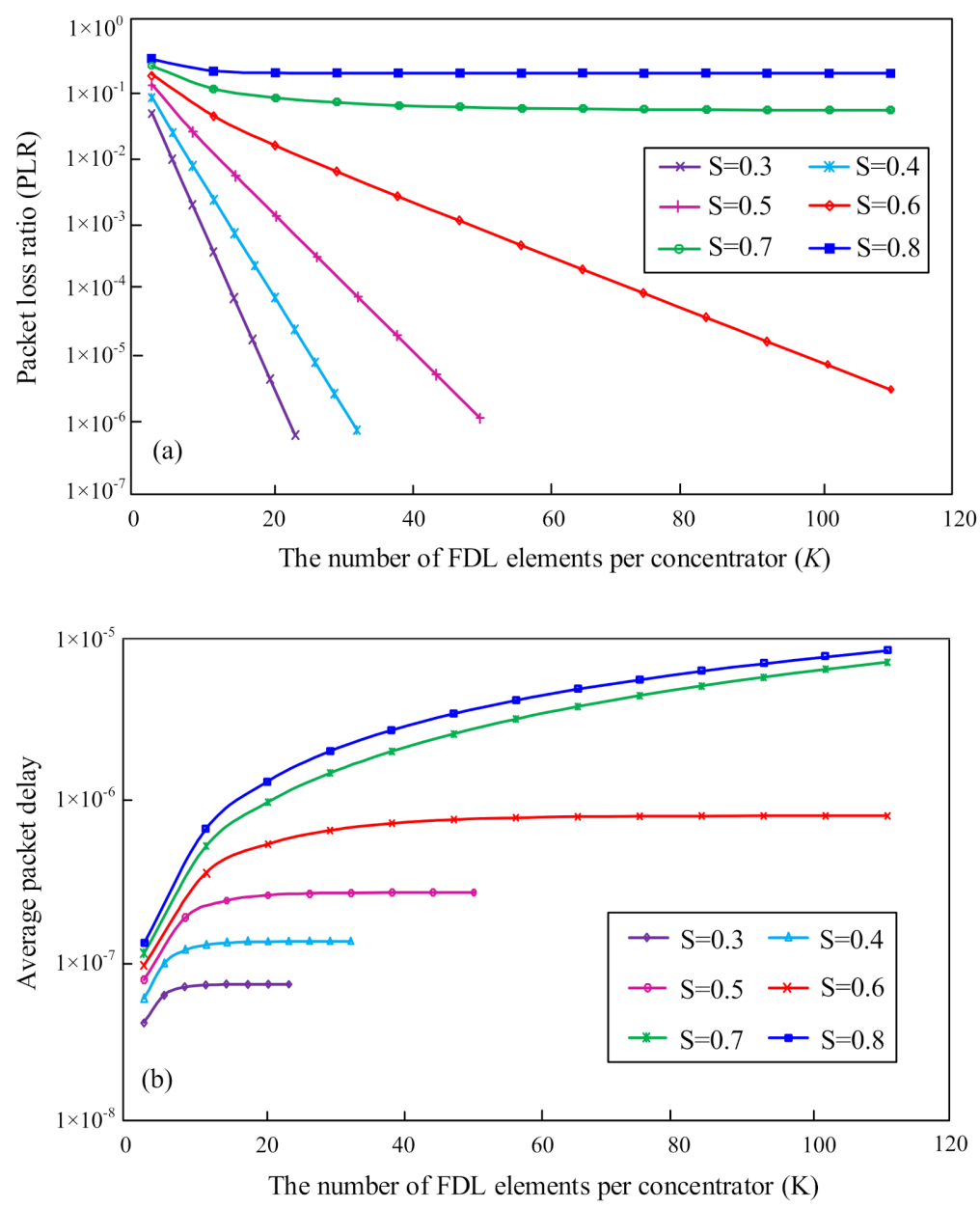

Figure 7. Packet loss ratio (PLR) and the average packet delay versus the buffer size offered per concentrator. (a) PLR versus buffer size per concentrator; (b) the average packet delay versus buffer size per concentrator.

Figure 7 shows that the total incoming traffic load arriving into each input concentrator should be smaller than 0.7 , for the considered case that the length of the basic FDL element is same as the mean packet length. For any higher incoming traffic load value, the buffer will become an overflow and the corresponding performance will deteriorate by increasing the buffer size, i.e., PLR cannot be improved while the delay time increases dramatically. 


\section{Discussions}

In this section we discuss the economic cost of the proposed OPS node compared with the classic OPS node without input concentrators.

In order to investigate the economic cost of the proposed OPS node architecture, the number of used optical gates (OGs) and TWCs are required, since both of which are expected to be the most expensive components in the proposed architecture. For the space switch of the input concentrator, a single state implementation is considered. The number of OGs is evaluated as the product between the number of inputs and outputs, i.e., $F \times(K+1)$ for each concentrator in Figure 2. Hence, the total number of OGs for the proposed OPS node is $F \times w \times(K+1)$, while no OG is used in the OPS node without input concentrators. Furthermore, the introduction of the input concentrator reduces the number of TWCs, which is foreseen as the most expensive in the switch. Correspondingly, the proposed OPS node only needs $w$ TWCs for handling the incoming traffic while the OPS node without input concentrators has to employ $F \times w$ TWCs.

Once the number of OGs and TWCs has been obtained, the cost comparison at the present time (indicated with $t=0$, time counter in year) can be carried out. The cost of an OG at the present year $\left(C_{\mathrm{OG}}(0)\right)$ is taken as the reference cost. The cost of a TWC is assumed to be $C_{T W C}(0)=\alpha C_{O G}(0)$, with $\alpha \gg 1$, since a TWC is undoubtedly much more complex and expensive. In the following, we use $C_{n}(0), C_{o}(0)$ to denote the proposed OPS node and the OPS node without input concentrators at the present time, which can be expressed as:

$$
\begin{gathered}
C_{n}(0)=F w(K+1) C_{O G}(0)+w C_{T W C}(0) \\
C_{o}(0)=F w C_{T W C}(0)
\end{gathered}
$$

The cost ratio $R$, which is defined as the economic cost ratio between the proposed OPS node with input concentrators and the OPS node without concentrators, can be obtained. The cost ratio at present time $t=0$ can be defined as:

$$
R(0)=\frac{C_{n}(0)}{C_{o}(0)}=\frac{F(K+1)+\alpha}{F \alpha}=\frac{K+1}{\alpha}+\frac{1}{F}
$$

In order to evaluate how the economic cost evolves as a function of time, the extended learning curve model in [18] is adopted. The model defines the cost evolution of a device " $X$ " $(X=\{O G, T W C\})$ in this paper $)$ and is given as:

$$
C_{X}(t)=C_{X}(0)\left\{n_{X}^{-1}\left[1+e^{\left(\ln \left(n_{X}^{-1}-1\right)-\frac{2 \ln 9}{\Delta T_{X}} t\right)}\right]^{-1}\right\}^{\log _{2} K_{X}}
$$

where the parameters are: $C_{X}(0)$, the production cost of the device in the reference year $t=0 ; n_{X}$, the relative accumulated volumes sold at the reference year; $\Delta T_{X}$, the growth period, which means the time for the accumulated volume to grow from $10 \%$ to $90 \%$ of the total production; $K_{X}$, the learning curve coefficient, which indicates the cost reduction when the production volume doubles. The values of those parameters for OG and TWC are assigned as in Table 2 according to the technology classification in [18]. We include the OGs into the New Fast Class (new components with technology improving fast) while TWC is included in the Emerging Medium and Emerging Slow classes, which are not already present in the market and expected to evolve in different ways. According to [18], $K_{X}$ is set as 0.8 for optical components and 0.7 for advanced optical components. Then $R(t)$ can be calculated according to its expression. 
Table 2. Values of parameters for OGs and TWCs.

\begin{tabular}{ccc}
\hline Parameters & OG & TWC \\
\hline$\Delta T_{X}$ & 5 & 20 \\
\hline$K_{X}$ & 0.8 & 0.7 \\
\hline$n_{X}$ & 0.01 & 0.001 \\
\hline
\end{tabular}

Figure 8 gives the cost ratio evaluation as a function of time (years) in case of $K=30$ and $\alpha=100$. Nine different OPS node configurations are considered. The main observation is that the cost ratio $R(t)$ is smaller than 1 in future 20 years, i.e., the proposed OPS node is much cheaper than the OPS node without input concentrators even 20 years into the future. Furthermore, as with the theoretical analysis, its evaluation only depends on the fiber number and is not affected by the wavelength number per fiber for fixed $K$ and $\alpha$. We also observe that $R(t)$ decreases as $F$ increases. This means that as the node degree grows (i.e., the number of input/output fibers per node grows), the proposed OPS architecture will have larger cost savings. In addition, $R(t)$ will diminish in the first 6 years and then increase for all nine configurations, as shown in Figure 8. This shows that, according to the cost evolution of TWCs and OGs, the advantage in the economic cost of the proposed OPS architecture will become more and more obvious in the next 6 years. However, as the development of the production technology for TWC, whose growth period is set as 20, it will become much cheaper as time goes on. Hence, the cost ratio will start to increase after 6 years. To conclude, the proposed OPS architecture has significant improvements compared with the OPS node without input concentrators due to the reduction of TWCs, the large cost saving at the present time for all considered configurations, and better cost performance in at least the next 20 years.

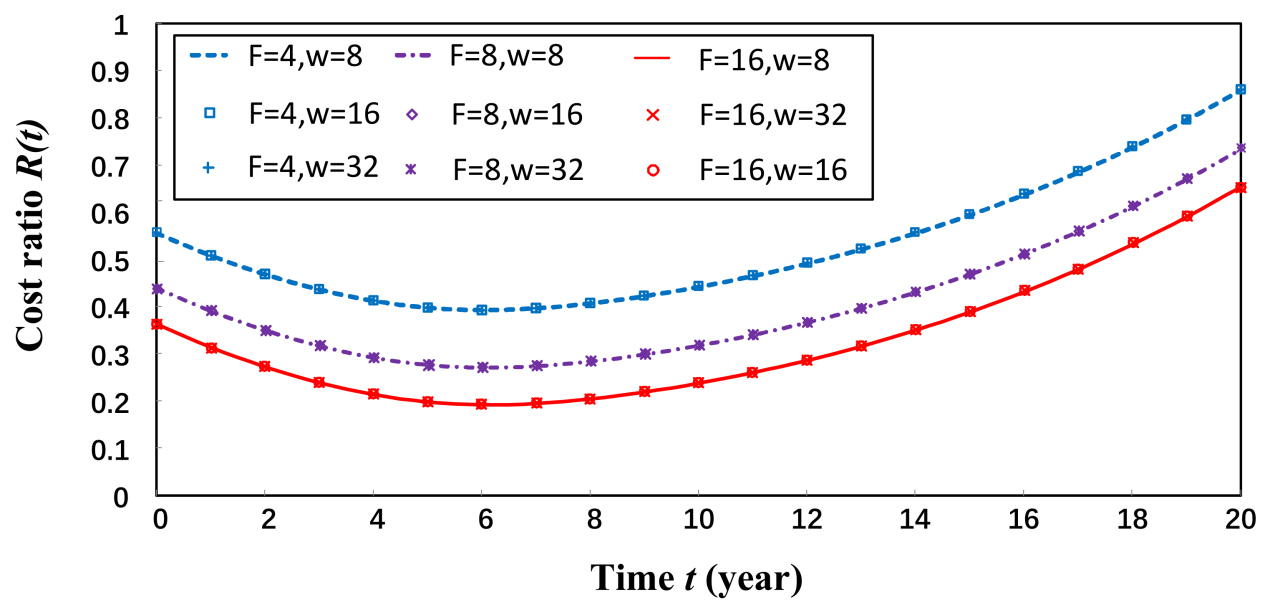

Figure 8. Cost ratio as a function of time in terms of years for different configurations in case of $K=30$ and $\alpha=100$.

\section{Conclusions}

In this paper, we propose a novel OPS architecture equipped with input concentrators, which is specially designed for the packet services with low bandwidth demand. The architecture and working principle of the OPS node for the arriving packets are described in detail. The given architecture of the concentrator can also be used for other networks, such as ORION and packet enhanced OTN. With the employment of input concentrators, the physical size and the economic cost of the total OPS node can be reduced significantly. Since the concentrators will introduce additional packet loss ratio (PLR) and delay time, a Markov model is built to analyze the performance of the proposed OPS architecture in terms of the PLR and the average packet delay. The corresponding closed form expressions are derived and given. This model demonstrates that the system performance can be 
greatly improved by increasing the buffer size when the total incoming traffic load is smaller than 0.69315. In order to evaluate the accuracy of the present model, we also built a discrete event simulator. The results show that the analytical model provides accurate PLRs and average packet delay for the studied OPS node under different network scenarios. In addition, the economic cost savings of the proposed OPS node at the present time, and the respective evolution as a function of time, are also discussed in detail.

Author Contributions: Conceptualization, H.Y. and X.M.; methodology, investigation, H.Y. and Z.F.; resources, X.M.; data curation, H.Y.; writing-original draft preparation, X.M.; writing-review and editing, Y.Z., W.C. and S.Y.; supervision, T.W., H.Y., S.Y. and H.C.; project administration, H.Y. and H.C. All authors have read and agreed to the published version of the manuscript.

Funding: This research was funded by the research project of State Grid Zhejiang Electric Power Co. Ltd., grant number 5211XT20008R.

Institutional Review Board Statement: Not applicable.

Informed Consent Statement: Not applicable.

Data Availability Statement: The data presented in this study are available on request from the corresponding author. The data are not publicly available as the data also forms part of an ongoing study.

Acknowledgments: The authors express their appreciation to the anonymous reviewers for their valuable suggestions.

Conflicts of Interest: The authors declare no conflict of interest.

\section{References}

1. Djordjevic, I.; Arabaci, M.; Minkov, L. Next Generation FEC for High-Capacity Communication in Optical Transport Networks. J. Light. Technol. 2009, 27, 3518-3530. [CrossRef]

2. Fiorani, M.; Casoni, M.; Aleksic, S. Hybrid Optical Switching for Energy-Efficiency and QoS Differentiation in Core Networks. J. Opt. Commun. Netw. 2013, 5, 484-497. [CrossRef]

3. Yao, L.; Nan, H.; Zheng, X. Fine-Grained all-optical switching based on optical time slice switching for hybrid packet-OCS intra-data center networks. In Proceedings of the IEEE Optical Fiber Communication Conference, Anaheim, CA, USA, 20-24 March 2016.

4. Gambini, P.; Renaud, M.; Guillemot, C.; Callegati, F.; Andonovic, I.; Bostica, B.; Chiaroni, D.; Corazza, G.; Danielsen, S.; Gravey, P.; et al. Transparent optical packet switching: Network architecture and demonstrators in the KEOPS project. IEEE J. Sel. Areas Commun. 1998, 16, 1245-1259. [CrossRef]

5. Zhu, X.; Kahn, J.M. Queueing models of optical delay lines in synchronous and asynchronous optical packet-switching networks. Opt. Eng. 2003, 42, 1741-1748. [CrossRef]

6. Sudbø, A.; Bjørnstad, S.; Cheyns, J.; Van Breusegem, E.; Zouganeli, E. Scalable optical switch structure based on tunable wavelength converters and arrayed waveguide grating routers. J. Opt. Netw. 2003, 2, 340-349.

7. Yang, S.; Stol, N.; Chi, H.; Li, Q. Optimized design of delay-line buffers with an input-feedback mechanism for asynchronous optical packet switching networks. Appl.Opt. 2016, 55, 8705-8712. [CrossRef] [PubMed]

8. Eramo, V.; Listanti, M. Wavelength converter sharing in a WDM optical packet switch: Dimensioning and performance issues. Comput. Netw. 2000, 32, 633-651. [CrossRef]

9. Eramo, V.; Listanti, M.; Nuzman, C.; Whiting, P. Whiting: Optical switch dimensioning and the classical occupancy problem. Int. J. Commun. Syst. 2002, 5, 127-141. [CrossRef]

10. Eramo, V.; Listanti, M. Input wavelength conversion in optical packet switches. IEEE Commun. Lett. 2003, 7, 281-283. [CrossRef]

11. Ahmadi, H.; Denzel, W.E.; Murphy, C.A.; Port, E. A high-performance switch fabric for integrated circuit and packet switching. Int. J. Commun. Syst. 2010, 2, 181-190. [CrossRef]

12. Hailu, D.H.; Lema, G.G.; Yekun, E.A.; Kebede, S.H. Unified study of Quality of Serive (QoS) in OPS/OBS networks. Opt. Fiber Technol. 2017, 36, 394-402. [CrossRef]

13. Bjornstad, S.; Hjelme, D.R.; Stol, N. A packet switched hybrid optical network with service guarantees. IEEE J. Sel. Areas Commun. 2006, 24, 97-107. [CrossRef]

14. Van Breusegem, E.; Cheyns, J.; De Winter, D.; Colle, D.; Pickavet, M.; Demeester, P.; Moreau, J. A broad view on overspill routing in optical networks: A real synthesis of packet and circuit switching? Opt. Switch. Netw. 2004, 1, 51-64. [CrossRef]

15. Cheng, M.; Jiang, M.; Zhu, J.; Yang, W. Status of Packet Enhanced Optical Transport Network Technology and Its Appli-cation in Metropolitan Area Network. Telecommun. Sci. 2013, 29, 127-131. (In Chinese) 
16. Vlachos, K.; Van Breusegem, E.; Christodoulopoulos, K.; Colle, D.; Ramantas, K.; Demeester, P. Constrained and Unconstrained overspill routing in optical networks: A detailed performance evaluation. Photon-Netw. Commun. 2007, 13, 227-240. [CrossRef]

17. Yao, S.; Mukherjee, B.; Dixit, S. Advances in photonic packet switching: An overview. IEEE Commun. Mag. 2000, 38, 84-94. [CrossRef]

18. Olsen, B.T.; Stordahl, K. Models for forecasting cost evolution of components and technologies. Telektronikk 2004, 4, 138-148. 